\title{
Performance Analysis Of FLS, EXP, LOG AND M-LWDF PACKET SCHEDULING ALgORITHMS IN DOWNLINK 3GPP LTE SYSTEM
}

\author{
Farhana Afroz ${ }^{1}$, Shouman Barua ${ }^{2}$, Kumbesan Sandrasegaran $^{2}$ \\ Faculty of Engineering and Information Technology, \\ University of Technology, Sydney, Australia
}

\begin{abstract}
Long-Term Evolution (LTE), an emerging and promising fourth generation mobile technology, is expected to offer ubiquitous broadband access to the mobile subscribers. In this paper, the performance of Frame Level Scheduler (FLS), Exponential (EXP) rule, Logarithmic (LOG) rule and Maximum-Largest Weighted Delay First (M-LWDF) packet scheduling algorithms has been studied in the downlink 3GPP LTE cellular network. To this aim, a single cell with interference scenario has been considered. The performance evaluation is made by varying the number of UEs ranging from 10 to 50 (Case 1) and user speed in the range of $[3,120] \mathrm{km} / \mathrm{h}$ (Case 2). Results show that while the number of UEs and user speed increases, the performance of the considered scheduling schemes degrades and in both case FLS outperforms other three schemes in terms of several performance indexes such as average throughput, packet loss ratio (PLR), packet delay and fairness index.
\end{abstract}

\section{KEYWORDS}

LTE, packet scheduling, QoS, FLS, M-LWDF, EXP rule, LOG rule

\section{INTRODUCTION}

The continuously increasing demand of real-time (RT) multimedia services along with high speed internet access and the need of having ubiquitous access to them even in high mobility scenarios are acting as a driver toward the evolution of wireless cellular networks. To keep pace with this rising demand, the Third-Generation Partnership Project (3GPP) introduced LTE which is also marketed as 4G mobile network. LTE network targets to provide high peak data rates (100 Mbps in downlink and $50 \mathrm{Mbps}$ in uplink within $20 \mathrm{MHz}$ bandwidth), spectrum flexibility (1.25 to 20 $\mathrm{MHz}$ ), improved system capacity and coverage, low user-plane latency (less than $5 \mathrm{~ms}$ ), high spectral efficiency, support of wide user mobility, reduced operating cost, enhanced support for end-to-end Quality of Service (QoS) and seamless interoperability with existing systems $[1,2]$.

In this context, effective utilization of radio resources becomes crucial. LTE radio access network (also known as E-UTRAN, Evolved-UMTS Terrestrial Radio Access Network) uses OFDMA (Orthogonal Frequency Division Multiple Access) radio access technology in downlink in which the available bandwidth is divided into parallel narrow-band orthogonal subcarriers with subcarrier spacing of $15 \mathrm{kHz}$ irrespective of total bandwidth and each UE is allocated with a set of subcarriers depending on user's requirements, existing system load, and the configuration of system [3]. E-UTRAN consists of eNBs only (the LTE terminology for base station) where all RRM (Radio Resource Management) functions such as physical layer functions, scheduling, admission control etc. are performed. Packet scheduling is the process by which available radio resources are allocated among active users in order to (re)transmit their packets so as the QoS

DOI : 10.5121/ijwmn.2014.6507 
requirements of the users are satisfied [4]. The main objectives of packet scheduling are to maximize the cell capacity, to satisfy the minimum QoS needs for the connections, and to maintain adequate resources for best-effort users with no strict QoS requirements [5]. LTE packet scheduling mechanism is not specified by $3 \mathrm{GPP}$, rather it is open for the vendors to implement their own algorithm. Different packet scheduling schemes has been proposed for LTE system. In this paper, the performance of FLS, LOG rule, EXP rule, and M-LWDF packet scheduling strategies has been studied by varying the number of users and users' speed.

The rest of this paper is organized as follows. A generalized packet scheduling model in the downlink LTE system is illustrated in section 2. Section 3 summarizes the dynamic packet scheduling schemes which were used in simulations followed by descriptions of the simulation scenarios and simulation results in section 4 . Finally, section 5 concludes the paper.

\section{Downlink Packet Scheduling Model}

In downlink LTE system, the smallest unit of radio resource that can be allocated to a user for data transmission is known as Physical Resource Block (PRB) which is defined both in time and frequency domain [5]. In the frequency domain, the total available bandwidth is split into 180 $\mathrm{kHz}$ sub-channels, each sub-channel corresponds to 12 consecutive and equally spaced subcarriers with sub-carrier spacing of $15 \mathrm{kHz}$ (i.e. each sub-channel is of $12 \times 15=180 \mathrm{kHz}$ ). In the time domain, the time is divided into frames and each LTE frame contains 10 consecutive TTIs (Transmission Time Interval). Each TTI is of $1 \mathrm{~ms}$ duration and consists of two time slots, each of $0.5 \mathrm{~ms}$ duration. Each time slot corresponds to 7 OFDM symbols (with short cyclic prefix). Resource allocation is performed on TTI basis. A time/frequency radio resource that spans over one time slot of $0.5 \mathrm{~ms}$ in the time domain and one sub-channel $(180 \mathrm{KHz})$ of 12 subcarriers in the frequency domain is known as Resource Block (RB). On every TTI, the RB pairs (in time domain) are allocated to a UE for data transmission.

The downlink packet scheduler aims to dynamically determine to which UE(s) to transmit packets and for each of the selected UE(s), on which Resource Block(s) (RB) the UE's Downlink Shared Channel (DL-SCH) will be transmitted [6]. A simplified packet scheduler model in LTE downlink system is shown in Fig. 1. In every TTI, each UE sends its CQI (Channel Quality Indicator) report computed from the downlink instantaneous channel condition to the serving eNB. At eNB, a buffer is assigned for each UE. Packets arriving at the buffer are time stamped and queued for transmission as FIFO (First In First Out) basis. On every TTI, scheduling decision takes place based on packet scheduling algorithms and one or more PRBs can be scheduled for each UE. There are specific scheduling criteria (e.g. channel condition, traffic type, head of line (HOL) packet delay, queue status etc.) for different scheduling strategies and depending on the scheduling criteria, users are prioritized. On each PRB, eNB choose a user with highest metric to transmit its packets. Once a user is selected, the number of bits transmitted per PRB depends on assigned Modulation and Coding Scheme (MCS) [7, 8]. 


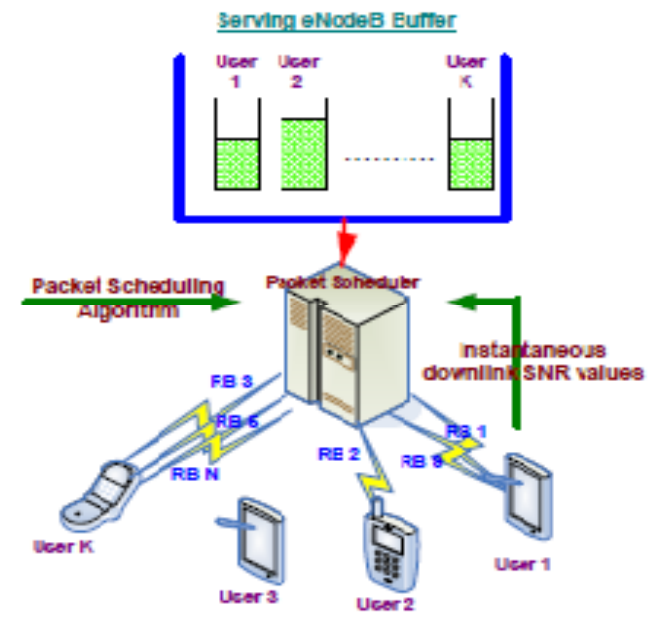

Fig. 1: A general LTE downlink packet scheduling model [8]

\section{Packet Scheduling Strategies}

LTE packet scheduling algorithm aims to maximize system performance. Different scheduling schemes have been proposed to support real-time (RT) and non real-time (NRT) applications. In this section, the algorithms that are considered in this paper will be described.

\subsection{Maximum-Largest Weighted Delay First (M-LWDF)}

M-LWDF [9] algorithm was proposed to support multiple real-time data users with different QoS requirements in CDMA-HDR system. A user is scheduled based on the following priority metric, M.

$M=\operatorname{argmax} a_{i} W_{i}(t) \frac{R_{i}(t)}{\bar{R}_{i}(t)}$

and $a_{i}=-\frac{\log \delta_{i}}{\tau_{i}}$

where $W_{i}(t)$ is the HOL packet delay of user $i$ at time $t, \tau_{\mathrm{i}}$ is the delay threshold of user $i$ and $\square_{i}$ denotes the maximum probability of HOL packet delay of user $i$ to exceed the delay threshold of user $i$.

Since, this scheme considers HOL packet delay together with PF properties, good throughput and fairness performance with a relatively low packet loss ratio (PLR) can be achieved using this algorithm.

\subsection{Frame Level Scheduler (FLS)}

This QoS (Quality of Service) aware packet scheduling algorithm was proposed in [10] for RT downlink communications. FLS is a two-level scheduling strategy where the two distinct levels (upper level and lower level) interact with each other to dynamically allocate RBs to the users. At upper level, a resource allocation scheme (namely FLS), which utilizes a D-T (Discrete-Time) linear control loop, is implemented. FLS specifies the amount of data packets that a RT source should transmit frame by frame to satisfy its delay constraint. At lower level, in every TTI, RBs are allocated to the UEs using Proportional Fair (proposed in [11]) scheme with taking into 
consideration the bandwidth requirements of FLS. Particularly, the scheduler at the lower layer defines the number of TTIs/RBs through which each RT source will send its data packets.

The amount of data to be transmitted is given by the following equation:

$v_{i}(k)=h_{i}(k) * q_{i}(k)$

Where, $v_{i}(k)$ is the amount of data to be transmitted by the $i$-th flow in $k$-th LTE frame, " $*$ " is the D-T convolution operator, $q_{i}(k)$ is the queue level. The above equation says that $v_{i}(k)$ is obtained by filtering the signal $q_{i}(k)$ through a time-invariant linear filter with pulse response $h_{i}(k)$.

\subsection{Exponential (EXP) Rule}

The Exponential rule [12], a channel aware/QoS aware scheduling strategy, was proposed to offer Quality of Service (QoS) guarantees to the users over a shared wireless link. It explicitly considers the channel conditions and the state of the queues while making scheduling decisions. The following two rules are called EXP rule.

The Exponential (Queue length) rule (EXP-Q) selects a single queue for service in time slot $t$

$i \in i(S(t))=\operatorname{argmax}_{i} \gamma_{i} \mu_{i}(t) \exp \left(\frac{a_{i} Q_{i}(t)}{\beta+[\bar{Q}(t)]^{\eta}}\right)$

where $\mu_{i}(t) \equiv \mu_{i}^{m(t)}$ and $\bar{Q}(t) \doteq\left(\frac{1}{N}\right) \sum_{i} a_{i} Q_{i}(t)$

Likewise, the Exponential (Waiting time) rule (EXP-W) selects for service a queue

$i \in i(S(t))=\operatorname{argmax}_{i} \gamma_{i} \mu_{i}(t) \exp \left(\frac{a_{i} W_{i}(t)}{\beta+[\bar{W}(t)]^{\eta}}\right)$

where $\bar{W}(t) \doteq\left(\frac{1}{N}\right) \sum_{i} a_{i} W_{i}(t)$

Here, $\gamma_{1}, \ldots \gamma_{N}$ and $a_{1} \ldots a_{N}$ are arbitrary set of positive constants, $\eta \in(0,1)$ is fixed and $\beta$ is positive constant. The EXP rule chooses either EXP-W or EXP-Q rule for service a queue.

\subsection{LOG Rule}

This channel aware/QoS aware strategy was designed to give a balanced QoS metrics in terms of robustness and mean delay [13]. Similar to the EXP rule, the scheduler allocates service to the user in a manner that maximizes current system throughput, with considering that traffic arrival and channel statistics are known. When users' queues are in state $\mathrm{q}$ and the channel spectral efficiencies of them are $\boldsymbol{K} \equiv\left(K_{i}: 1 \leq i \leq N\right)$, LOG rule scheduler serves a user $\mathrm{i}_{\mathrm{LOG}}$ :

$i_{L O G}(q, K) \in \arg \max _{1 \leq i \leq N} b_{i} \log \left(c+a_{i} Q_{i}\right) \times K_{i}$

Here, $b_{i}, a_{i}, c$ are fixed positive constants, $0<\eta<1$ and $Q_{i}$ represents the queue length.

\section{Performance Evaluation}

The performance evaluation of FLS, EXP rule, LOG rule and M-LWDF scheduling schemes with increasing number of UEs (Case 1) and varying UE's speed (Case 2) will be reported in this 
section. To this aim, an open source simulator namely LTE-Sim [14] has been adopted. LTE-Sim simulator exploits Jain's fairness method [15] to calculate fairness index among UEs. The propagation loss model includes the following:

-Fast fading: Jakes model

-Path loss: $L=128.1+37.6 \log 10 d @ 2 \mathrm{GHz}$, where $\mathrm{d}$ is the distance between user and $\mathrm{eNB}$ in $\mathrm{Km}$ -Penetration loss: 10dB

-Shadow fading: Lognormal distribution with mean 0 and standard deviation $8 \mathrm{~dB}$

\subsection{Case 1: Effects of number of users}

The performance of FLS, EXP rule, LOG rule, and M-LWDF downlink packet scheduling schemes with increasing the number of UEs is analyzed herein. For multimedia flows, the considered scheduling schemes have been compared based on several performance metrics named average throughput, PLR, delay, and the fairness index. For best effort (BE) flows, since there is no strict QoS requirements, a comparison among these scheduling strategies is reported on the basis of average throughput only.

\subsubsection{Simulation scenario}

A single urban macro cell with interference simulation scenario with each UE having single flow (video or VoIP or BE) and 40\% UEs receiving video flows, $40 \%$ users receiving VoIP flows and the rest $20 \%$ receiving $\mathrm{BE}$ flows has been taken into consideration to study the effects of number of users on the performance of the scheduling strategies described above. A number of UEs ranging from 10 to 50 are uniformly distributed and moving with a speed of $120 \mathrm{~km} / \mathrm{h}$ in random direction within a cell. Table 1 shows the simulation parameters.

Table 1. Simulation parameters

\begin{tabular}{|l|l|}
\hline Parameters & Value \\
\hline Simulation time & $150 \mathrm{sec}$ \\
\hline Cell radius & $1 \mathrm{Km}$ \\
\hline User speed & $120 \mathrm{~km} / \mathrm{h}$ \\
\hline Video bit rate & $242 \mathrm{kbps}$ \\
\hline Frame structure & FDD \\
\hline Bandwidth & $10 \mathrm{MHz}$ \\
\hline Flow duration & $120 \mathrm{sec}$ \\
\hline Maximum delay & $0.1 \mathrm{sec}$ \\
\hline
\end{tabular}

\subsubsection{Results and Discussion}

The average throughput graphs of video, VoIP and best effort flows in Fig. 2 demonstrate that the average throughput degrades while the number of users increases and FLS algorithms shows best average throughput performance for multimedia flows. As seen in Fig. 2(a), the average throughput of video flow falls upon increasing number of users for all the considered scheduling algorithms. For FLS algorithm, while the number of users increases from 10 to 20, the average throughput sharply falls followed by a steady decline in average throughput when the cell is charged with more than 20 users. M-LWDF and LOG rule provides almost identical throughput performance and EXP rule shows higher average throughput than these two schemes. The average throughput per VoIP flow (shown in Fig. 2(b)) maintains almost the constant level at 3000 bps in 
the user range of 10 to 40 for all four schemes. When the user number exceeds 40 , the average throughput slowly drops for all four schemes with increasing users. These no-variation trend of VoIP average throughput may be due to the VoIP traffic model (ON/OFF Markov chain) and the ON/OFF periods used during simulation. The average throughput graph of best effort flow in Fig. 2(c) depicts that while the user number increases, LOG rule and M-LWDF provide better average throughput performance compared with FLS algorithm whereas, EXP-rule provides higher average throughput than FLS scheme for the users ranging from 20 to 50.

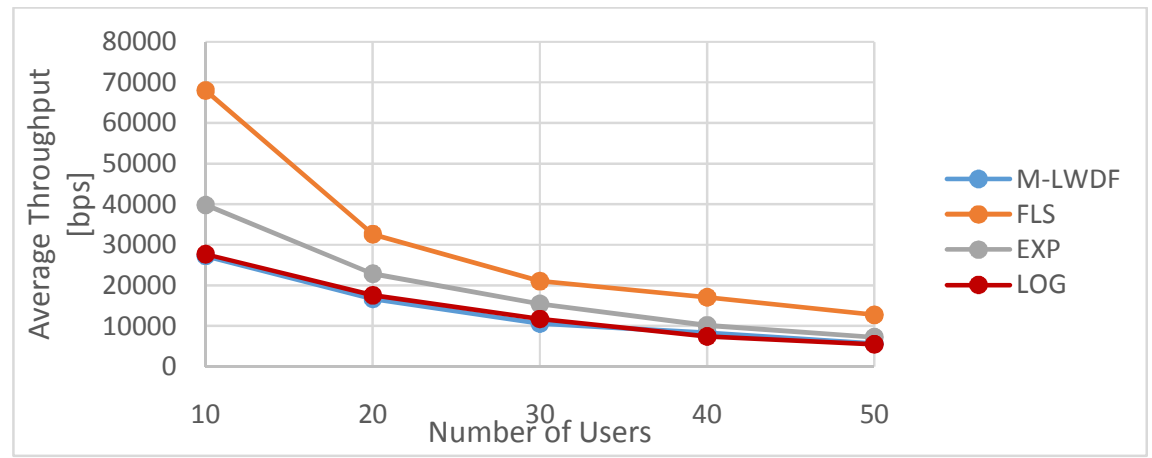

(a)

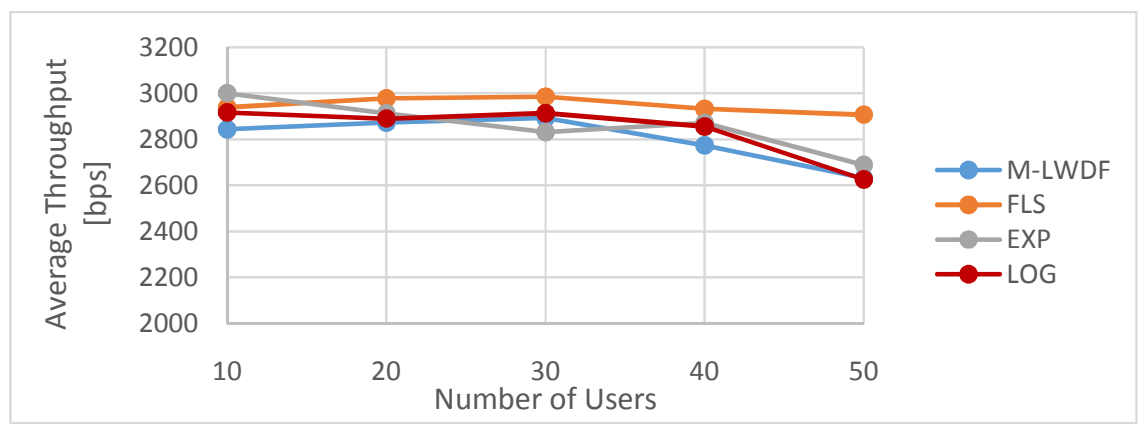

(b)

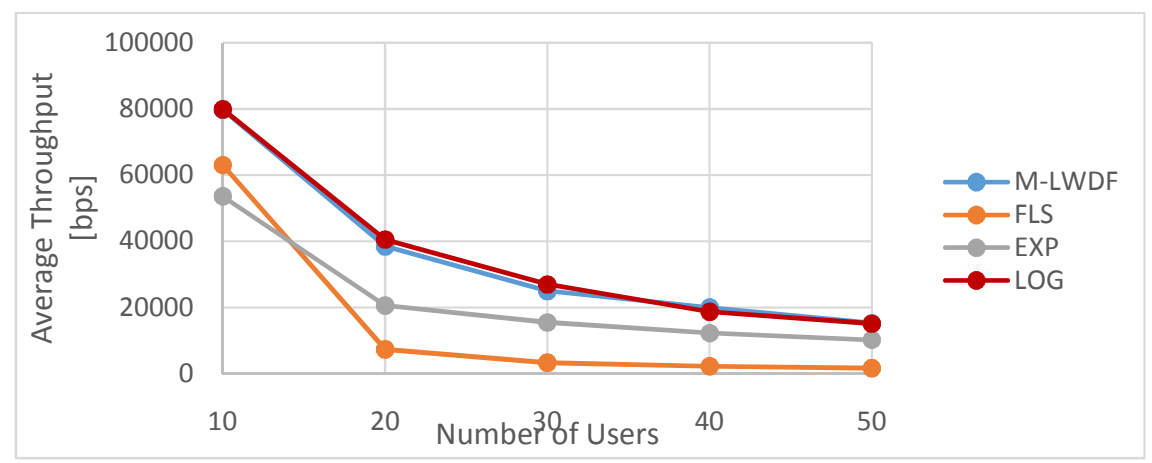

(c)

Fig. 2: Average throughput of (a) video flow (b) VoIP flow (c) BE flow

Fig. 3, showing the packet loss ratio (PLR) experienced by video and VoIP flows, describes that the PLR increases with increasing number of users because of increased network loads and the PLRs experienced by VoIP flows are considerably smaller than that of video flows for all four 
scheduling schemes. It can be also noticed that for multimedia flows, lowest PLRs are achieved using FLS algorithm and EXP rule offers better performance (i.e. smaller PLR) as compared with LOG rule and M-LWDF. As seen in Fig. 3(a), for video flow, LOG rule and M-LWDF provide almost same PLR performance. From Fig. 3(b), it is noticed that for VoIP flow, FLS algorithm maintains below $1 \%$ of PLR in the user range of 10 to 50. The PLRs remain within 5\% for LOG rule and M-LWDF scheme and within 3\% for EXP rule in the range of 10-40 users.

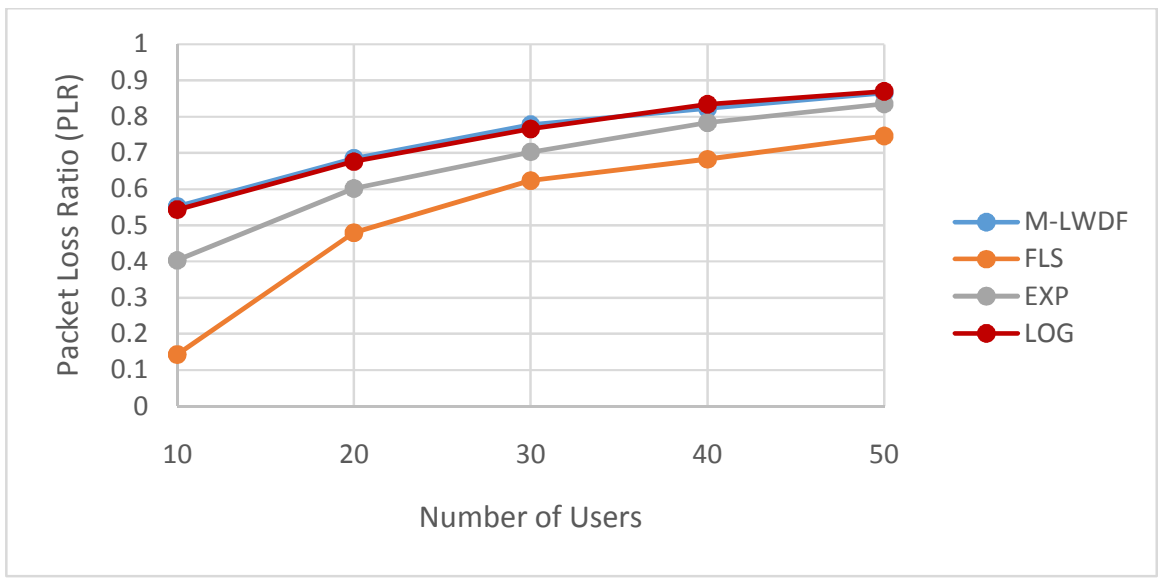

(a)

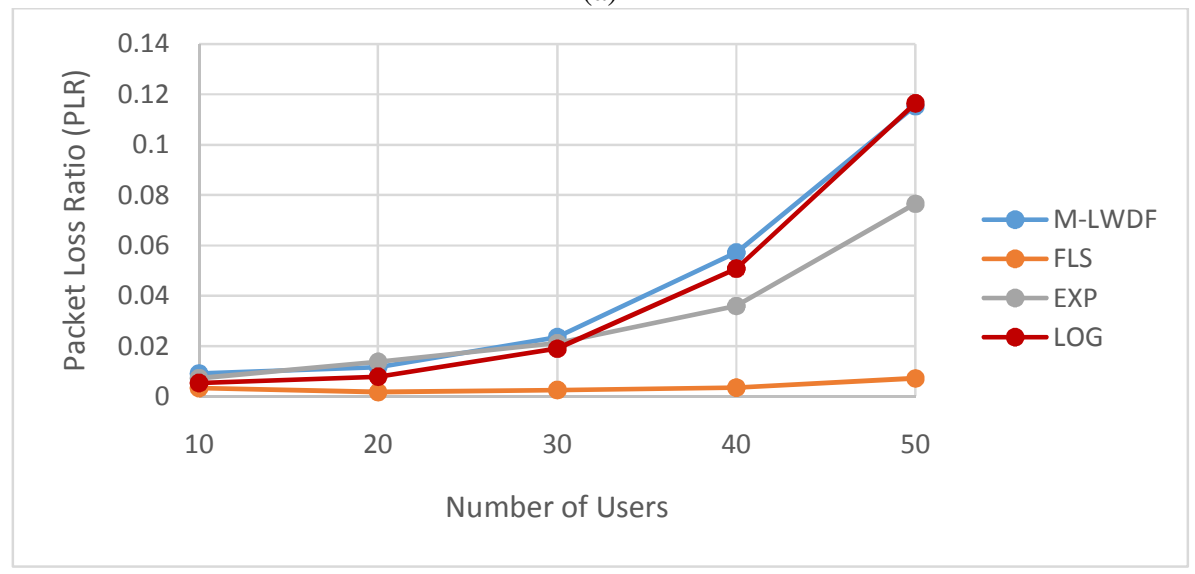

(b)

Fig. 3: PLR of (a) video flow (2) VoIP flow 
International Journal of Wireless \& Mobile Networks (IJWMN) Vol. 6, No. 5, October 2014

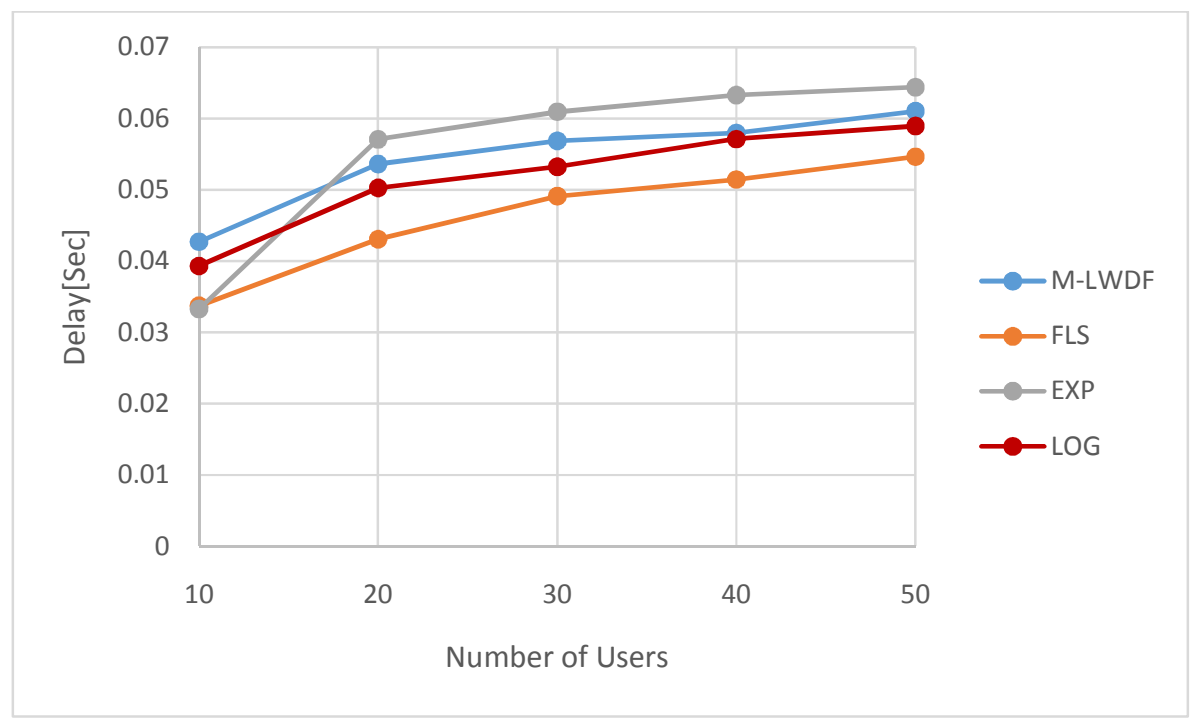

(a)

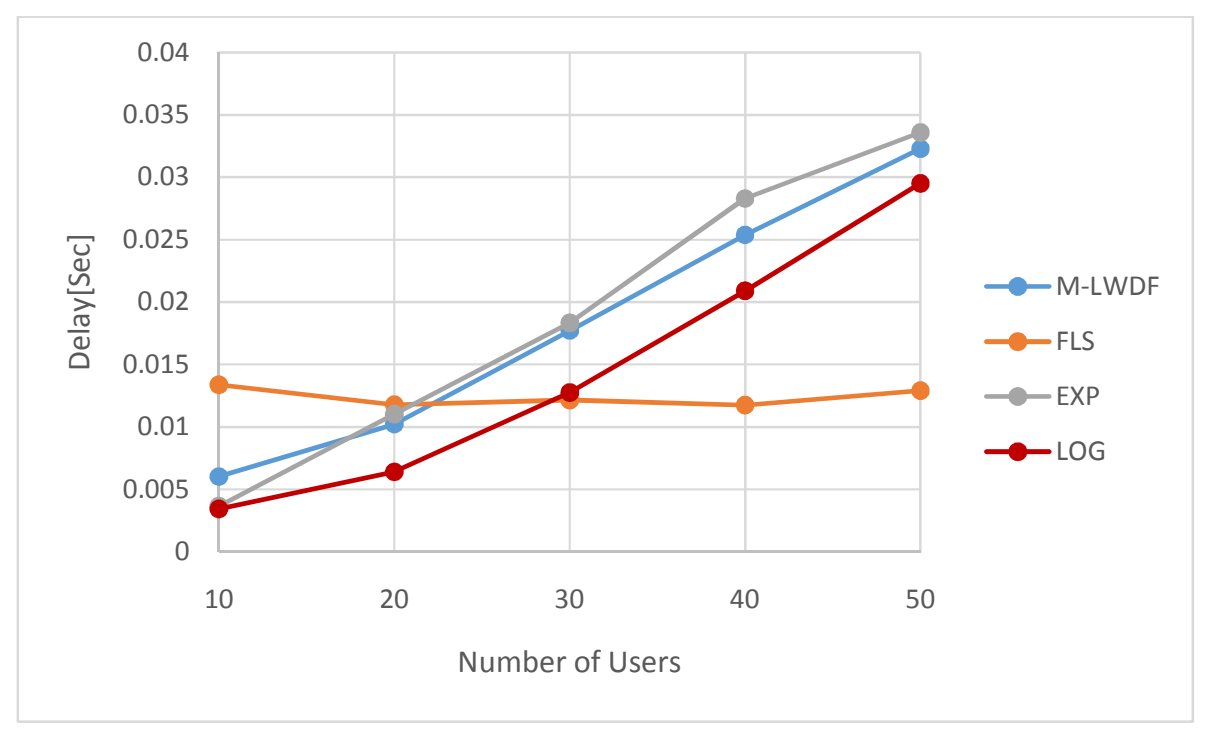

(b)

Fig. 4: Packet delay of (a) video flow (b) VoIP flow

As seen in Fig. 4(a), the packet delay of video flow gradually increases with increasing number of users for all four schemes and FLS is showing lowest delay among them. Fig. 4(b) showing the packet delay of VoIP flow illustrates that, for FLS scheme the packet delay maintains almost same level while increasing number of users. It is observed that FLS is giving lowest upper bound of the delay among four schemes and hence shows the lowest PLR.

Fig. 5(a) illustrates that for video flow, fairness index degrades with increasing number of users for all the four algorithms and FLS scheme ensures highest degree of fairness among them. In case of VoIP flow (Fig. 5(b)), fairness indexes are maximum when the cell is charged with 10 users and minimum when the user number is 50 for all four scheduling schemes with FLS is having the highest fairness index. 
International Journal of Wireless \& Mobile Networks (IJWMN) Vol. 6, No. 5, October 2014

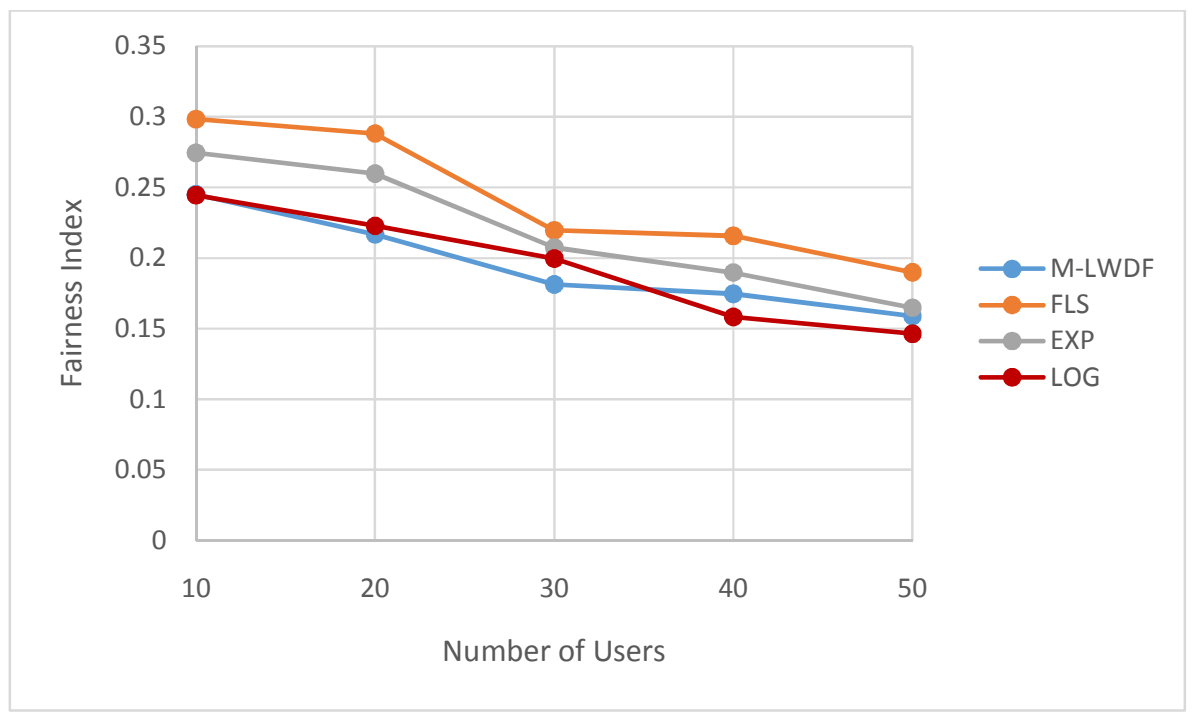

(a)

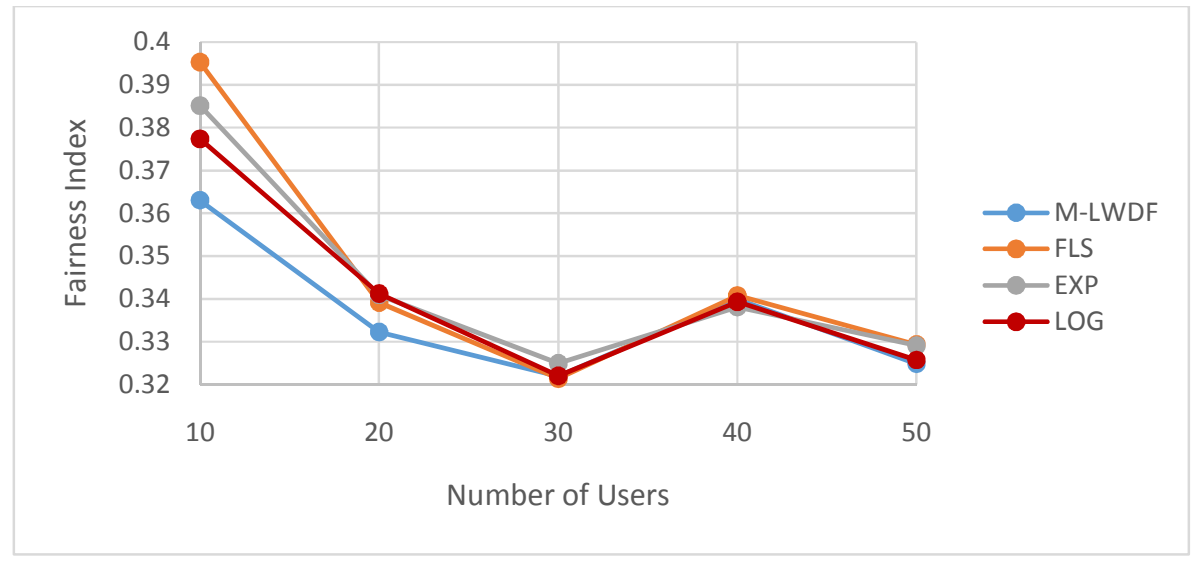

(b)

Fig. 5: Fairness index of (a) video flow (b) VoIP flow

\subsection{Case 2: Effects of users' speed}

In this part, two distinct user speed (pedestrian speed $-3 \mathrm{~km} / \mathrm{h}$ and vehicular speed $-120 \mathrm{~km} / \mathrm{h}$ ) are considered to study the effects of user's speed on the performance of the FLS, EXP rule, LOG rule and M-LWDF packet scheduling algorithms.

\subsubsection{Simulation scenario}

The simulation scenario considered here is identical to that of Case 1 (Subsection 4.1.1). The simulation parameters are given in Table 2. 
Table 2. Simulation parameters

\begin{tabular}{|l|l|}
\hline Parameters & Value \\
\hline Simulation time & $150 \mathrm{sec}$ \\
\hline Cell radius & $1 \mathrm{Km}$ \\
\hline User speed & $3 \mathrm{~km} / \mathrm{h}$ and $120 \mathrm{~km} / \mathrm{h}$ \\
\hline Video bit rate & $242 \mathrm{kbps}$ \\
\hline Frame structure & FDD \\
\hline Bandwidth & $10 \mathrm{MHz}$ \\
\hline Flow duration & $120 \mathrm{sec}$ \\
\hline Maximum delay & $0.1 \mathrm{sec}$ \\
\hline
\end{tabular}

\subsubsection{Results and Discussion}

Fig. 6 illustrates the effects of user speed on the average throughputs of BE flow, video flow and VoIP flows. As seen, the average throughputs of video flow (as seen in Fig. 6(a)) and BE flow (Fig. 6(c)) decrease with increasing users' speed from $3 \mathrm{~km} / \mathrm{h}$ to $120 \mathrm{~km} / \mathrm{h}$ for all four schemes. It is expected that average throughput decrease with increasing user speed because at higher speed channel quality measured by UE becomes worse, which in turn triggers lower order modulation to be selected and thus results in lower average throughput. From the graph of VoIP average throughput (Fig. 6(b)), it is observed that for FLS, the average throughputs of VoIP flow maintains almost the same level while the user speed increases. For EXP rule, LOG rule, MLWDF, the VoIP average throughput degrades with increasing user speed at higher speed. The packet loss ratios (PLRs) of video flow and VoIP flow, reported in Fig 7(a) and 7(b) respectively, show that for multimedia flows, the PLRs become greater when the users are at higher speed. The reason is- at higher speed poor link adaptation occurs. As seen in Fig. 8, the packet delay increases with increasing user speed for all four schemes. Fig. 9(a) demonstrates that, for video flow the fairness index falls at higher user speed for all four algorithms and FLS provides higher degree of fairness at both user speed. It is seen from the Fig. 9(b) that for VoIP flow, the considered scheduling schemes provide approximately same fairness index irrespective of user speed.

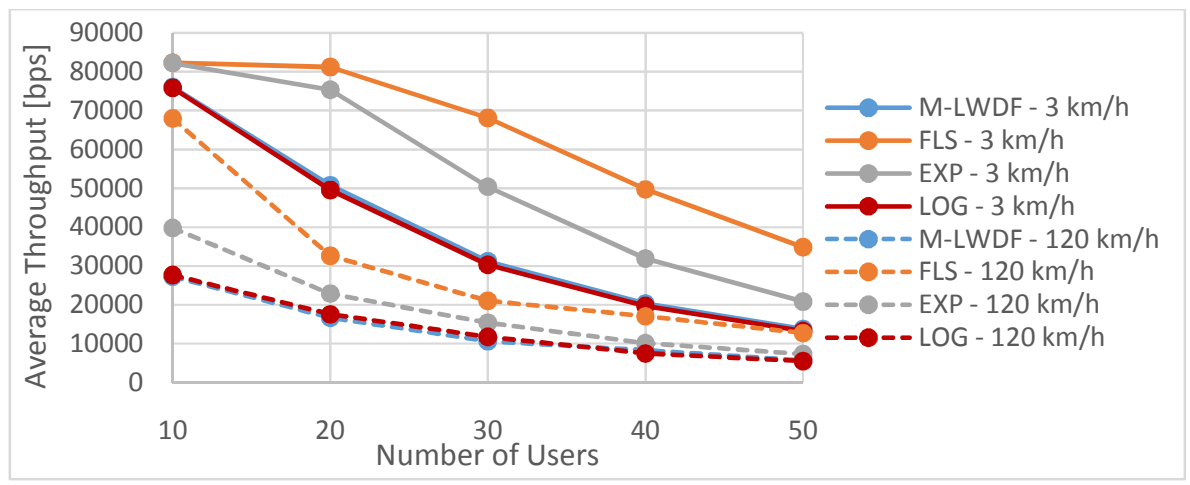

(a) 
International Journal of Wireless \& Mobile Networks (IJWMN) Vol. 6, No. 5, October 2014

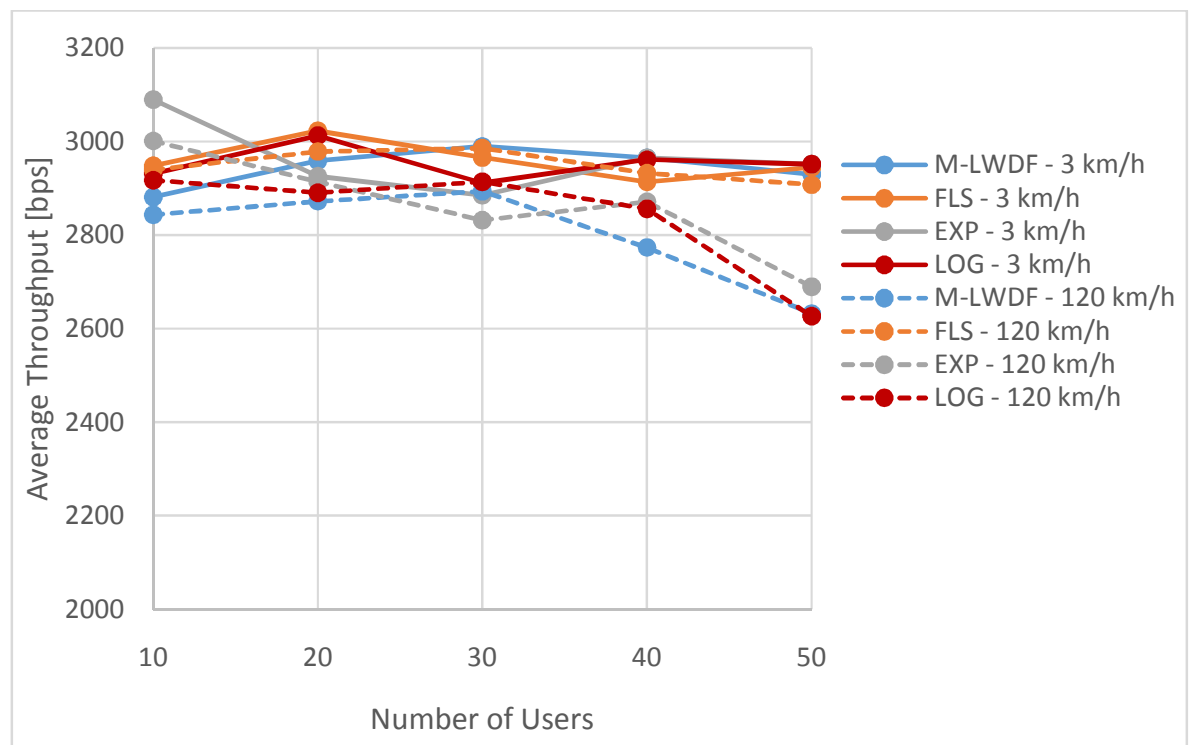

(b)

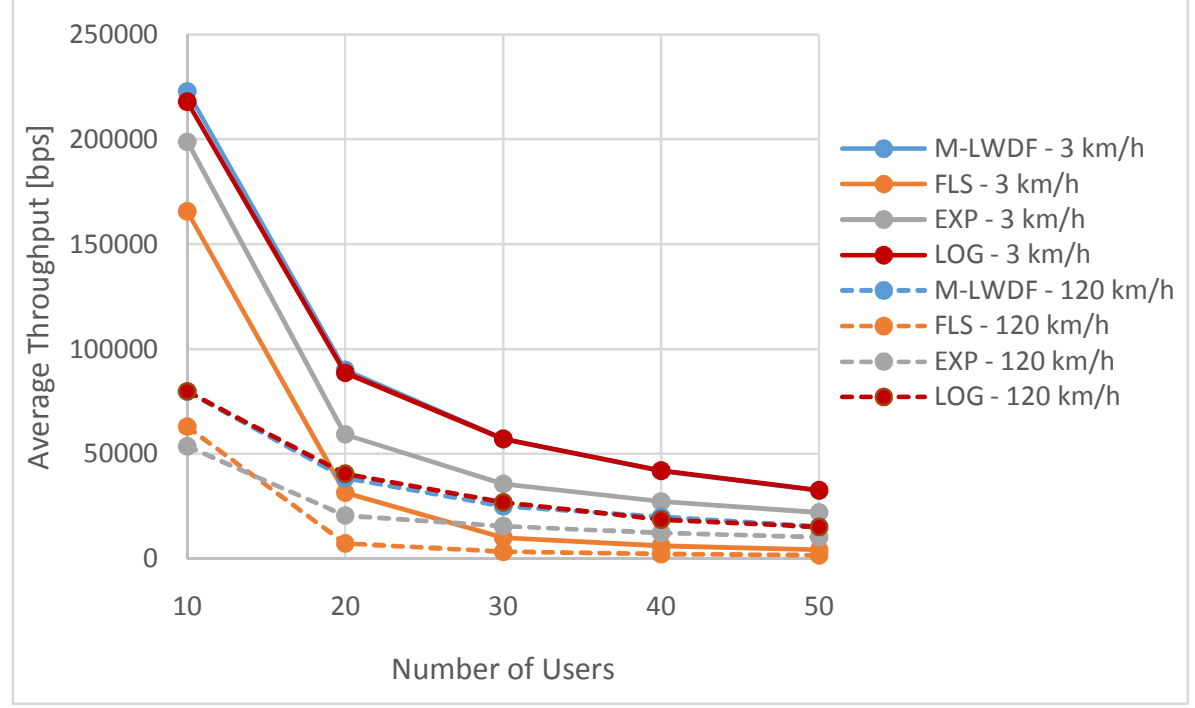

(c)

Fig. 6: Average throughput of (a) video flow (b) VoIP flow (c) BE flow 
International Journal of Wireless \& Mobile Networks (IJWMN) Vol. 6, No. 5, October 2014

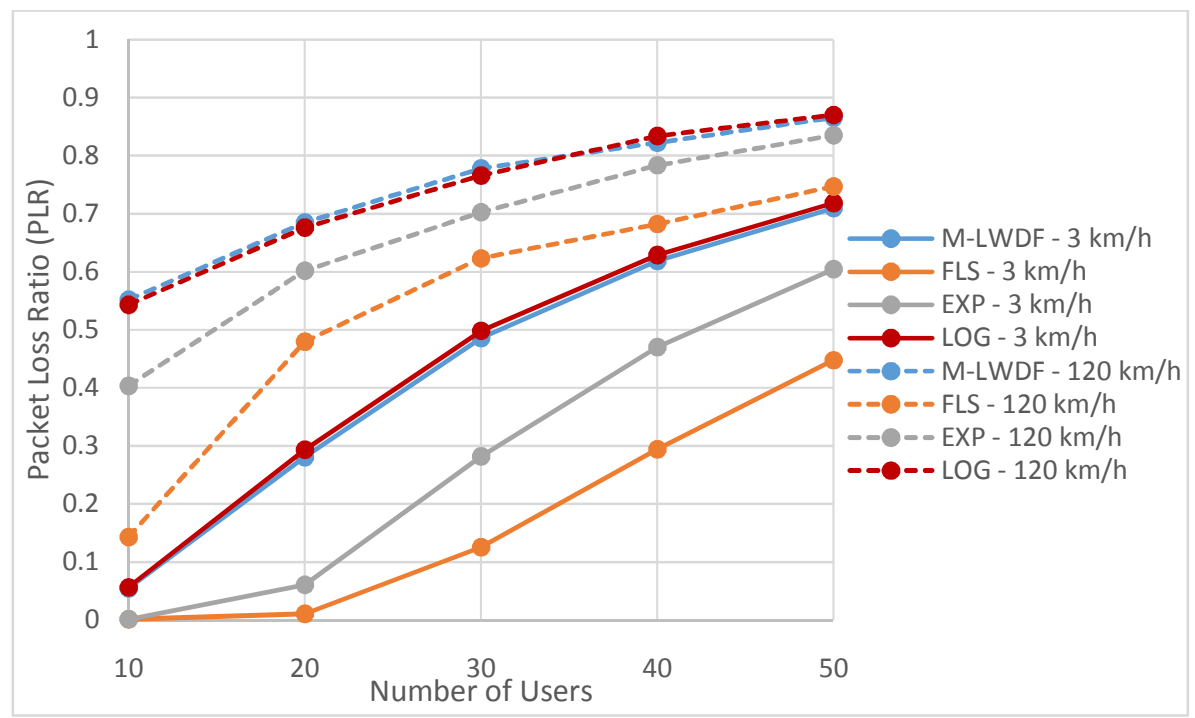

(a)

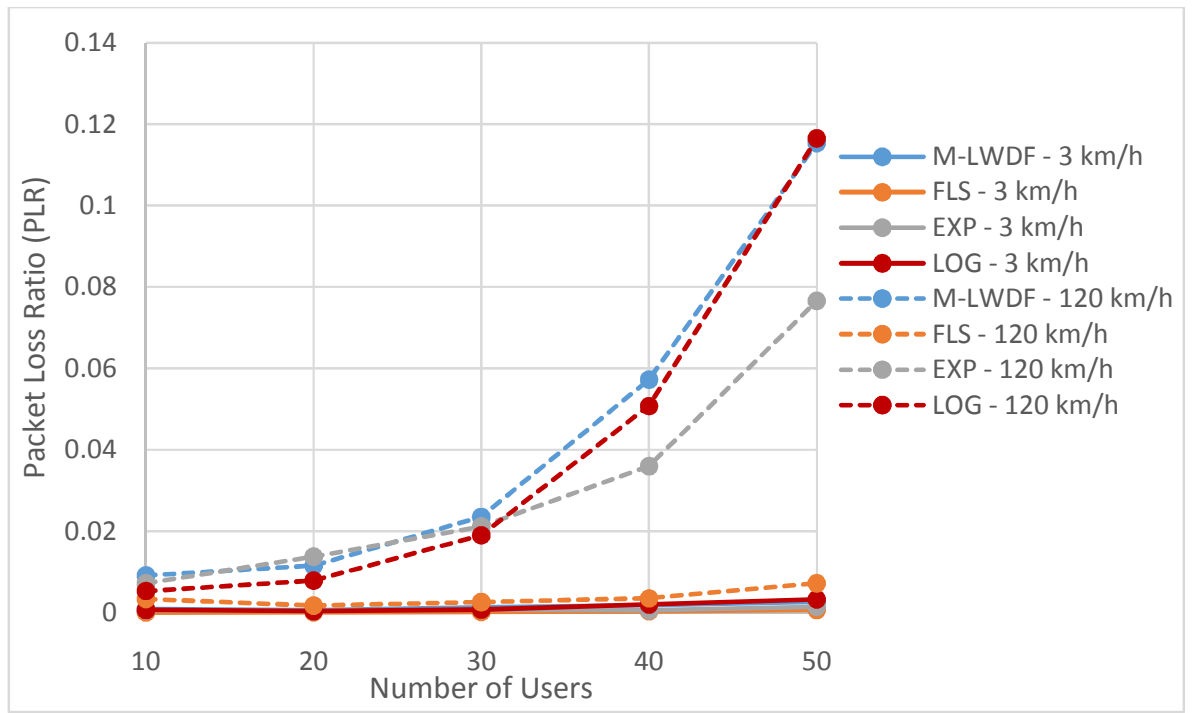

(b)

Fig. 7: PLR of (a) video flow (b) VoIP flow 
International Journal of Wireless \& Mobile Networks (IJWMN) Vol. 6, No. 5, October 2014

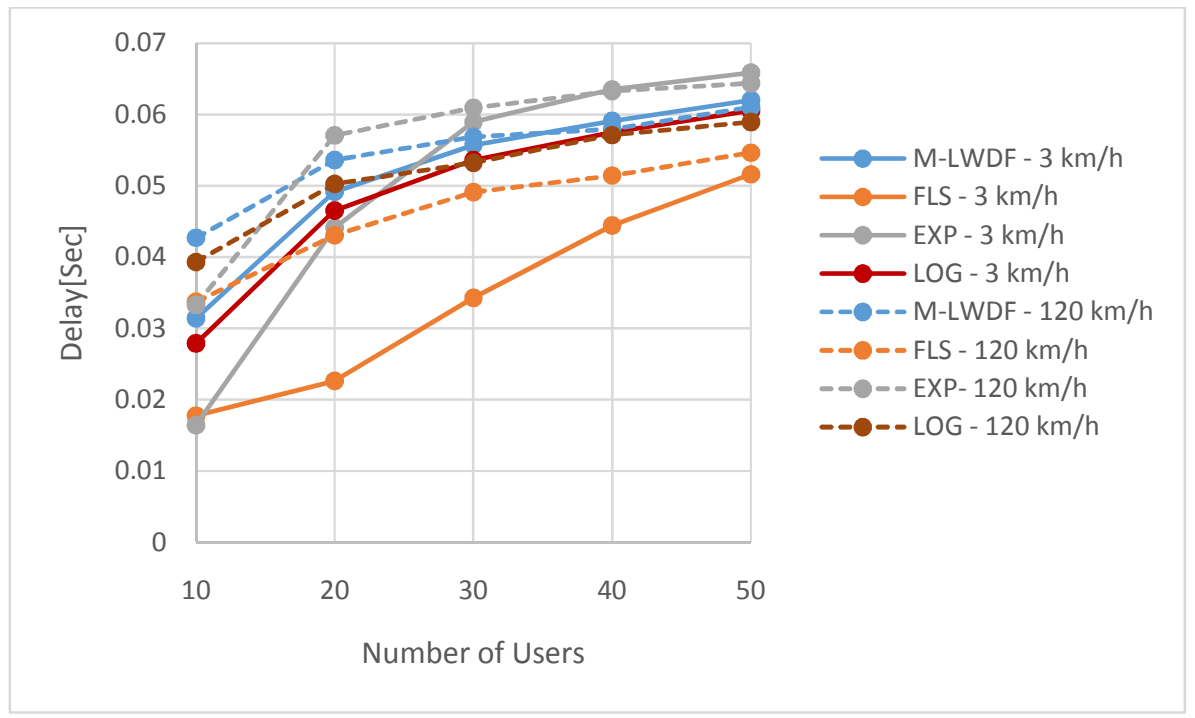

(a)

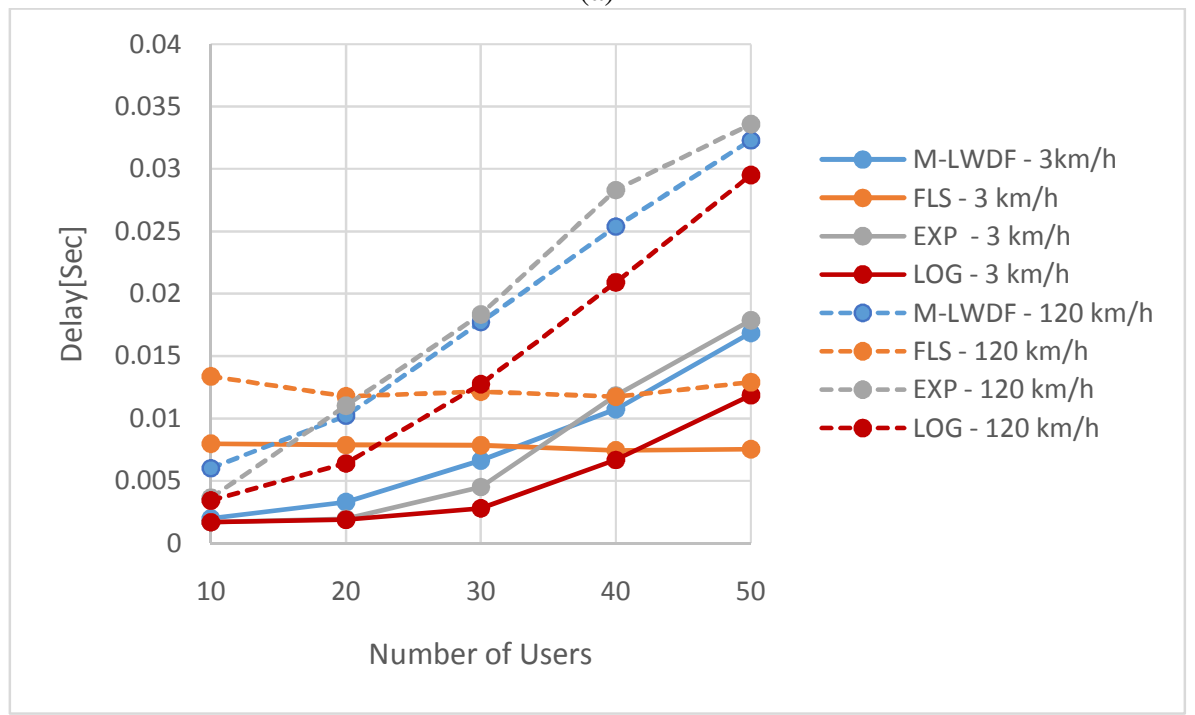

(b)

Fig. 8: Packet delay of (a) video flow (b) VoIP flow 
International Journal of Wireless \& Mobile Networks (IJWMN) Vol. 6, No. 5, October 2014

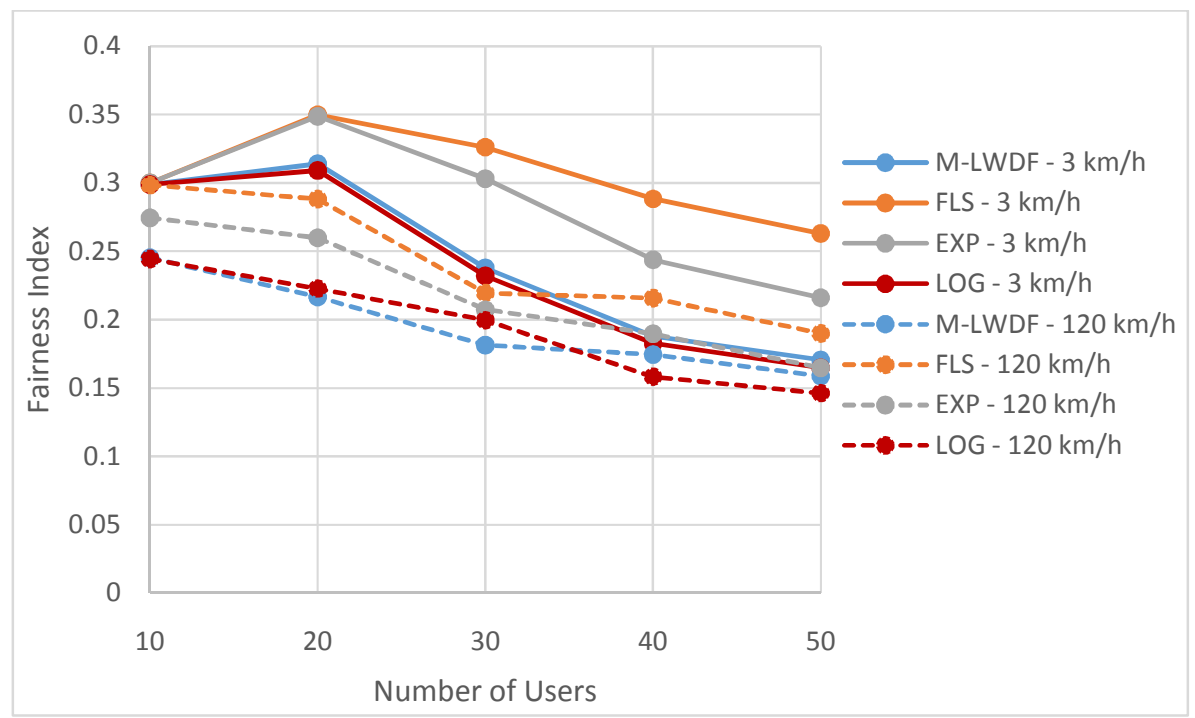

(a)

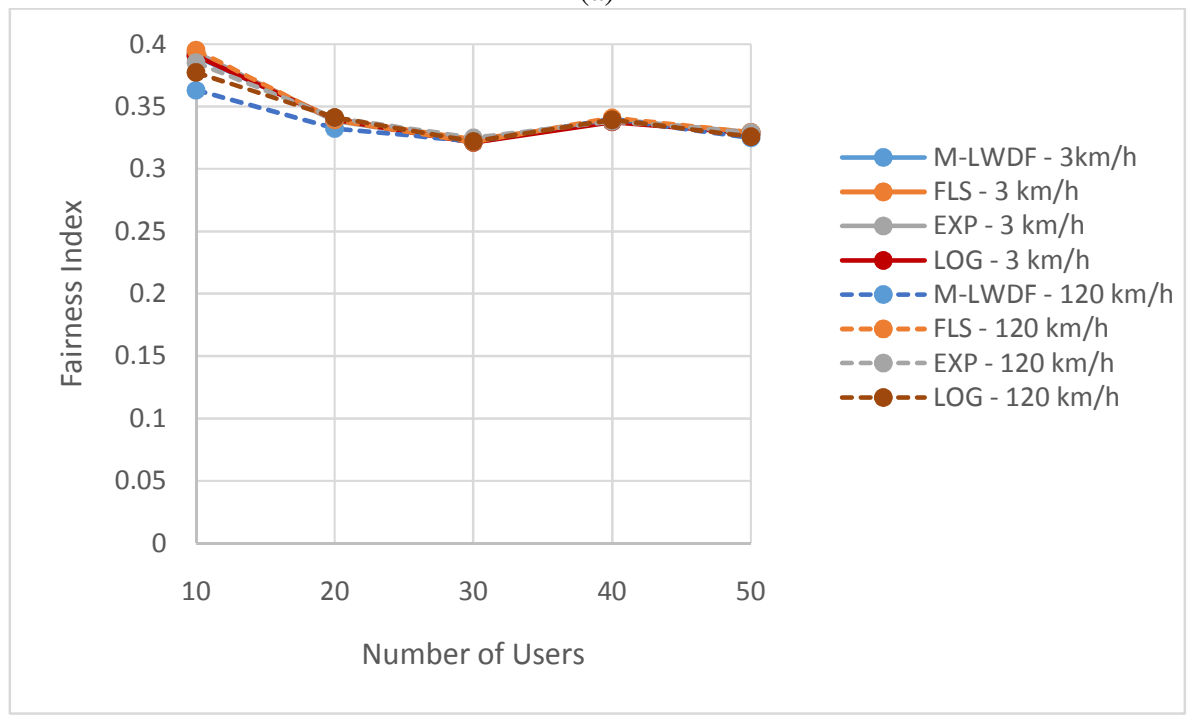

(b)

Fig. 9: Fairness index of (a) video flow (b) VoIP flow

\section{CONCLUSION}

In this paper, the performance study of FLS, EXP rule, LOG rule and M-LWDF packet scheduling algorithms in LTE downlink has been performed while varying number of users and users' speed. The simulation results show that overall FLS scheme outperforms other three schemes in terms of average throughput, PLR, delay, and fairness index. It is also reported that the performance of simulated packet scheduling strategies drops noticeably while the users' speed increases. Our future work includes to simulate and compare the performance of LTE downlink packet scheduling algorithms with different scenarios. 
International Journal of Wireless \& Mobile Networks (IJWMN) Vol. 6, No. 5, October 2014

\section{REFERENCES}

[1] A. Ghosh and R. Ratasuk, Essentials of LTE and LTE-A: Cambridge University Press, 2011.

[2] 3GPP Technical Report, TR 25.913, "Requirements for Evolved UTRA (E-UTRA) and Evolved UTRAN (E-UTRAN)," version 7.0.0, June 2005.

[3] H.A.M. Ramli, K. Sandrasegaran, R. Basukala, L. Wu, "Modeling and Simulation of Packet Scheduling in the Downlink Long Term Evolution System," 15th Asia-Pacific Conference on Communications, Oct. 2009, pp.68-71.

[4] G. Piro, L. A. Grieco, G. Boggia, F. Capozzi, and P. Camarda, "Simulating LTE cellular systems: an open- source framework," IEEE Transactions on Vehicular Technology, Feb. 2011, vol. 60, pp. 498513.

[5] H. Holma and A. Toskala, LTE for UMTS : OFDMA and SC-FDMA based radio access, Chichester, U.K.: Wiley, 2009.

[6] E. Dahlman, S. Parkvall, and J. Sköld, 4G LTE/LTE-Advanced for Mobile Broadband: Academic Press, 2011.

[7] R. Basukala, H. A. M. Ramli, and K. Sandrasegaran, "Performance analysis of EXP/PF and M-LWDF in downlink 3GPP LTE system," 1st AH-ICI on internet, Nov. 2009, pp. 1-5.

[8] H. A. M. Ramli, R. Basukala, K. Sandrasegaran, and R. Patachaianand,"Performance of well known packet scheduling algorithms in the downlink 3GPP LTE system," in Communications (MICC), IEEE 9th Malaysia International Conference , Dec. 2009, pp. 815-820.

[9]M. Andrews, K. Kumaran, K. Ramanan, A. Stolyar, P. Whiting, and R. Vijayakumar, "Providing Quality of Service over a Shared Wireless Link," IEEE Communications Magazine, vol. 39, pp. 150$154,2001$.

[10] Giuseppe Piro, Luigi Alfredo Grieco, Gennaro Boggia, Rossella Fortuna, and Pietro Camarda, (2011) "Two-Level Downlink Scheduling for Real-Time Multimedia Services in LTE Networks", IEEE Transaction on Multimedia, Vol. 13, No. 5.

[11] A. Jalali, R. Padovani, and R. Pankaj, "Data Throughput CDMAHDR a High Efficiency-High Data Rate Personal Communication Wireless System," in IEEE 51st Vehicular Technology Conference Proceedings, Tokyo, 2000, pp. 1854-1858.

[12] S. Shakkottai and A. Stolyar, (2002) "Scheduling for Multiple Flows Sharing a Time-Varying Channel: The Exponential Rule", Analytic Methods in Applied Probability, Vol. 207 of American Mathematical Society Translations, Series 2, A Volume in Memory of F. Karpelevich, pp. 185202, American Mathematical Society, Providence, RI, USA.

[13] B. Sadiq, S.J.Baek, and G. de Veciana, (2009) "Delay-Optimal Opportunistic Scheduling And Approximations: The Log rule", Proceedings of the 27th Annual Joint Conference on the IEEE Computer and Communications Societies (INFOCOM 09), pp. 19.

[14] G. Piro, L. A. Grieco, G. Boggia, F. Capozzi and P. Camarda, (2011) "Simulating LTE Cellular Systems: An Open-Source Framework”, IEEE Transaction on Vehicular Technology, Vol. 60, No. 2.

[15] R. Jain, D. Chiu, and W. Hawe, A quantitative measure of fairness and discrimination for resource allocation in shared computer systems, Digital Equip. Corp., Littleton, MA, DEC Rep., DEC-TR-301, Sep. 1984. 